\title{
DESCRIPCIÓN DE DOS ESPECIES NUEVAS DE ACHROMOPORUS (DIPLOPODA: POLYDESMIDA: CHELODESMIDAE) PARA LA REPÚBLICA DOMINICANA*
}

\author{
Carlos Suriel
}

Museo Nacional de Historia Natural de Santo Domingo (MNHNSD). Calle César Nicolás Penson, Plaza de la Cultura, Santo Domingo, República Dominicana. c.suriel@museohistorianatural.gov.do

\section{RESUMEN}

Se describen dos especies nuevas del género Achromoporus Loomis, 1936, elevándose a 26 el número de especies de este género para La Hispaniola. Se presentan ilustraciones de los gonopodos y del dorso de ambas especies. Los especímenes fueron colectados en localidades de la provincia Peravia, República Dominicana.

Palabras clave: Achromoporus, Chelodesmidae, Diplopoda, especies nuevas, provincia Peravia, República Dominicana, La Hispaniola.

\section{ABSTRACT}

Two new species of the genus Achromoporus Loomis, 1936, are described from the Dominican Republic, increasing to 26 the number of species of this genus from Hispaniola. Illustrations of gonopods and dorsum are presented. The specimens were collect in Peravia Province, Dominican Republic.

Key words: Achromoporus, Chelodesmidae, Diplopoda, new species, Peravia Province, Dominican Republic, Hispaniola.

\section{INTRODUCCIÓN}

Una reciente revisión del género Achromoporus Loomis, 1936, incluyendo la descripción de 11 especies nuevas y la sugerencia de varias sinonimias, elevó a 22 su número de especies (Pérez-Asso, 2009). Poco después se adicionan otras dos especies (Suriel, 2010), destacándose que este género pasaba a ser el más diverso dentro de la familia Chelodesmidae en La Hispaniola. Ahora agregamos que es uno de los táxones más interesantes para futuros estudios en sistemática, biogeografía y biología evolutiva. Con las dos descripciones que se presentan en esta oportunidad, se eleva a 26 el número de especies de este género conocidas en la isla.

Los quelodésmidos, al igual que otras familias de la clase Diplopoda, siguen ofreciendo especies nuevas en cada expedición que realiza el Museo Nacional de Historia Natural de Santo Domingo. Otras especies de Achromoporus aún están pendientes de descripción en nuestros laboratorios. La percepción compartida es que, por mucho tiempo, los bosques dominicanos seguirán ofreciéndonos sorpresas con su interesante fauna de milpiés.

\footnotetext{
*Este trabajo ha sido posible gracias a las expediciones realizadas en el marco de dos proyectos de estudio financiados por FONDOCYT, del Ministerio de Educación Superior, Ciencia y Tecnología (MESCyT) de la República Dominicana, correspondientes a los números 2008-2-C2-040 y 2009-102. Ambos proyectos son dirigidos por el Instituto de Investigaciones Botánicas y Zoológicas "Rafael M. Moscoso" de la Universidad Autónoma de Santo Domingo (UASD) y cuentan con la responsabilidad compartida del Museo Nacional de Historia Natural de Santo Domingo.
} 


\section{OBJETIVO}

- Describir dos especies nuevas para la ciencia, correspondientes al género Achromoporus Loomis, 1936; Familia Chelodesmidae; Orden Polydesmida; Clase Diplopoda (milpiés).

\section{MATERIALES Y MÉTODOS}

Los especímenes fueron colectados en Las Yayitas, Loma Las Yayas, Loma Los Pinos y Loma Los Guayuyos (provincia Peravia). Una vez colectados, fueron fotografiados vivos y luego preservados en alcohol etílico al 70\%. Las observaciones se realizaron con un microscopio estereoscópico MEIJI TECHNO modelo EMZ-5TR. Los dibujos fueron realizados por el autor; para los rasgos generales del dorso y los gonopodos completos se usaron fotos tomadas con una cámara Nikon, modelo Coolpix 4500, adaptada a un microscopio estereoscópico marca Leica, modelo MZ7.5. Las imágenes se procesaron con el programa Auto Montage versión 5.0; los detalles se precisaron con el material al microscopio. Para los dibujos de las piezas gonopodales separadas se utilizó una cámara lúcida marca LEITZ WETZLAR. Los dibujos fueron escaneados y editados con Photoshop versión CS5 e Ilustrator CS5, para su corrección. Para las mediciones se usaron una Miniescala de Bio Quip Products, Inc. modelo "Métrica" con un rango de $5 \mathrm{~mm}$ y divisiones de $0.1 \mathrm{~mm}$, así como una regla milimetrada transparente.

Para la nomenclatura taxonómica se siguió a Hoffman (1979). Las medidas del cuerpo se tomaron siguiendo a Pérez-Asso (1996): longitud, desde el extremo de la cabeza hasta el ápice del epiprocto, y la anchura entre los ángulos posteriores de los paraterguitos en el segmento 7. La medida que se ofrece en cada caso corresponde a la media, indicándose entre paréntesis el rango de variación y el número de ejemplares. Las coordenadas geográficas y la altitud de las localidades se registraron usando un GPS marca Garmin, modelo ETREX.

Abreviaturas: MNHNSD (Museo Nacional de Historia Natural de Santo Domingo), SX ( $\mathrm{S}=$ diplosegmento, $\mathrm{X}=$ número del diplosegmento correspondiente); APP (ángulo posterior del paraterguito) y AAP (ángulo anterior del paraterguito), en Inglés: PPA (paranota posterior angle) y PAA (paranota anterior angle), respectivamente. ARPA (Colección Privada Antonio R. Pérez-Asso).

\section{RESULTADOS}

\section{CLASIFICACIÓN}

Familia Chelodesmidae Cook, 1895.

Subfamilia Chelodesminae Hoffman, 1979.

Género Achromoporus Loomis, 1936 (sensu Pérez-Asso, 2009).

Referencias consideradas. Achromoporus Loomis, 1936, Bull. Mus. Comp. Zool., 80 (1):112119; 1941, Bull. Mus. Comp. Zool., 88 (2): 17-80. Jeekel, 1971, Monog. Nederl. Entom. Vereng., 5, pp.1-412. Hoffman, 1979, Mus. Hist. Nat. Genéve: 153, pp. 209; 1999, Virg. Mus. Nat. Hist. Spec. Public., 8: pp. 288. Pérez-Asso, 2005, Solenodon, 5: 53-59; 2009, Solenodon, 8: 33-81. Pérez-Asso y Pérez-Gelabert, 2001, Bol. S.E.A., 28: 73-74. Pérez-Gelabert, 2008, ZOOTAXA 1831, 71, pp. 530. Suriel, 2010, Novitates Caribaea, 3, 13-21.

Achromoporus bastardoae sp. nov.

Figura 1, A-E

Diagnosis. Gonopodos con acropodito expandido laminalmente, retorcido en porción inferior (fig. 1, C). Bifurcación en porción terminal, rama posterior semiespatulada, ligeramente más 
ancha, rama anterior (solenomerito) filiforme en el extremo (fig. 1, C-D). Proceso prefemoral expandido y acanalado en su cara externa, extremo apiculado hacia dentro (fig. 1, E). Prominencia cónica en coxas del segundo par de patas del macho. Hipoprocto subtriangular, valvas anales ligeramente estriadas. Patas rojizas. Cuerpo ligeramente aplanado en región anterior, ligeramente globoso en región posterior (fig. 1, A-B), sobre todo en hembras. Color castaño-rojizo, más claro en el centro del margen posterior de metaterguitos, abarcando prozonitos, formando en esta parte manchas en forma de uno o dos lunares claramente notorios en especímenes vivos. Dorso sin setas. No hay diferencia de color entre segmentos poríferos y no poríferos. Ausencia de dientes en paraterguitos, con margen posterior liso. APP blancos (fig. 1, A), con márgenes engrosados en segmentos no poríferos, reducidos en región anterior, no inclinados hacia atrás, excepto ligeramente en últimos segmentos (fig. 1, B). Callos poríferos presentes, los poros abren lateralmente. Fórmula porífera normal (5, 7, 9-10, 12-13, 15-19).

Diagnosis. Gonopods with acropodite bifurcated at upper portion, wide and laminated, twisted at lower portion, with small folds at middle portion (fig. 1, C). Posterior branch slightly wider and slightly spatulated, the anterior branch (solenomere) distally filiform (fig.1, C-D). Prefemoral process wide, with channel on external face, acute apex, curves inward (fig. 1, E). Coxae of second pair of legs with conical process in the males. Hypoproct subtriangular, anal valves slightly striated. Legs reddish. Body slightly flattened at anterior portion, slightly convex at posterior portion (fig. 1, A-B), mainly in females. Color reddish chestnut, light chestnut at the middle of posterior margin of metaterga, including prozonites, forming at this portion one or two spots like stains clearly evident in living specimens. Dorsum glabrous. Poriferous and nonporiferous segments with similar color pattern. Paranota dentation absent, it's posterior margin smooth. Posterior corner of paranota (PPA) white (fig. 1, A), with thickened margin at nonporiferous paranota, short at anterior body, inclined lightly backwards at posterior segments (fig. 1, B). Peritremata (callus) present, ozopore opening laterally. Pore formula normal (5, 7, 9-10, 12-13, 15-19).

Descripción del Holotipo. Macho (ð̋). Número catalográfico: MNHNSD19.1,590. Longitud= $26.0 \mathrm{~mm}$, anchura $=3.8 \mathrm{~mm}$.

Gonopodos. Largos, sobrepasan margen posterior de S6. Acropodito muy retorcido en porción inferior, expandido laminalmente, la bifurcación en porción terminal, las dos ramas son cortas y parecidas; rama posterior más ancha que rama anterior, semiespatulada; rama anterior filiforme en su extremo, el cual es curvado hacia atrás. Proceso prefemoral expandido desde su porción inferior, acanalado en su cara externa, terminado en extremo agudo, apiculado y doblado hacia dentro (fig. 1, C-E).

Vista dorsal. Color castaño rojizo, más claro en margen posterior de metaterguitos, con mancha rojiza más clara en forma de semicírculo en el centro de este y el pleurito. Forma del cuerpo y color de segmentos como en Diagnosis. Las antenas extendidas alcanzan el margen posterior de S3, antenómeros gruesos, aproximadamente del mismo tamaño, últimos más pubescentes. APP blanquecino en todos los segmentos, coloración más pronunciada en segmentos poríferos, agudo en su extremo, sobretodo en segmentos posteriores. Nódulos muy pequeños en paraterguitos a partir de S2, insinuados; estos forman dos hileras paralelas más arriba del margen posterior del metaterguito en segmentos S17-S19. Fórmula porífera normal (5,7, 9-10, 12-13, 15-19). Callo porífero presente. Epiprocto ligeramente elongado, pares de macrosetas visibles (fig. 1, B).

Vista ventral. Pleuritos color castaño, más oscuros que esternitos. Proceso cónico pequeño en coxas del segundo par de patas. Las patas son rojizas. Hipoprocto subtriangular, del mismo color que esternitos; valvas anales más oscuras, ligeramente estriadas. 


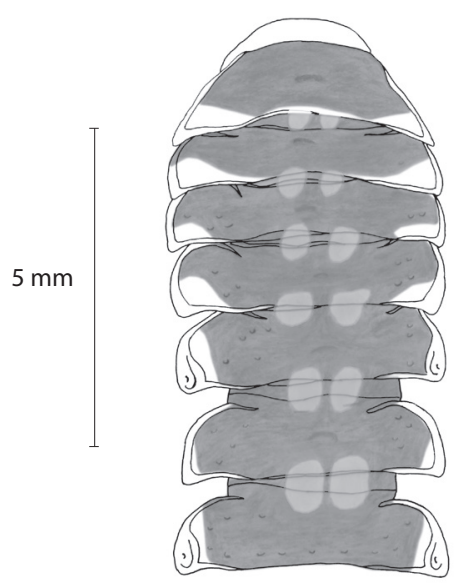

A

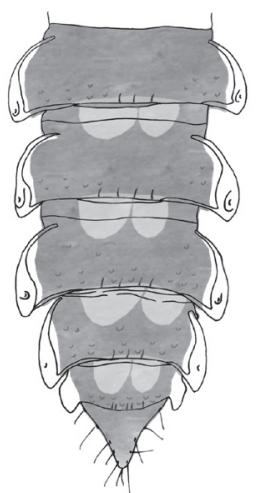

B
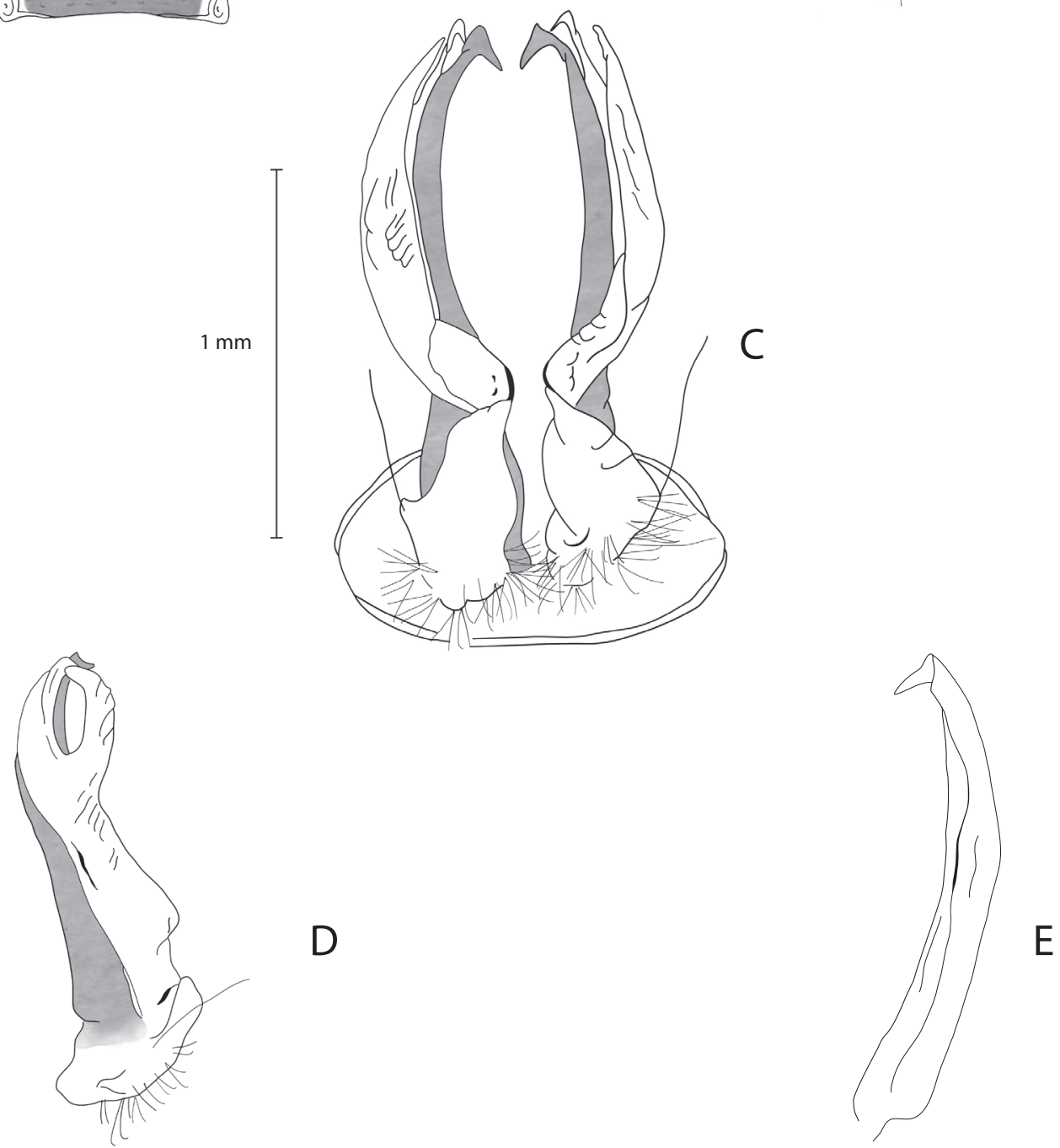

Figura 1. Partes del cuerpo y gonopodos de Achromoporus bastardoae sp. nov. A, región anterior del cuerpo. B, región posterior del cuerpo. C, gonopodos completos, vista posterior (proceso prefemoral sombreado para diferenciar). D, rama gonopodal derecha, girada para mostrar bifurcación del acropodito (proceso prefemoral sombreado). E, proceso prefemoral. A-C, holotipo, macho 19. 1,590. D-E, paratipo, macho 19. 1,587. 
Variabilidad y regularidades morfológicas. Machos: longitud $=26.6 \mathrm{~mm}(24.0-30.5 ; \mathrm{n}=12)$, anchura $=3.92 \mathrm{~mm}(3.5-4.6 ; \mathrm{n}=12)$. Hembras: longitud $=29.6 \mathrm{~mm}(27.9-33.2 ; \mathrm{n}=14)$, anchura $=4.54 \mathrm{~mm}(4.0-5.5 ; \mathrm{n}=14)$.

En general, las hembras de las diferentes localidades presentan cuerpo menos aplanado que los machos, con la región posterior ligeramente más globosa. APP ligeramente más corto y menos puntiagudo, con la coloración blanquecina menos notable. Los machos de la localidad Loma Los Pinos también presentan APP más corto y menos puntiagudo, en comparación con los machos de la localidad tipo.

Gonopodos. No se aprecian diferencias en la estructura gonopodal entre los machos.

Vista dorsal. No se observan diferencias de color entre segmentos poríferos y segmentos no poríferos. La coloración en terguitos es menos pronunciada en ejemplares de Loma Los Pinos $(\lesssim ð 1,450,717$ y 718) y de Loma Las Yayas, Honduras (ð714). APP con color blanco más pronunciado en segmentos poríferos, pero menos notable en hembras, más corto y menos puntiagudo en ejemplares de Loma Los Pinos; se observó el color blanco de APP menos pronunciado en algunos segmentos de $\widehat{\jmath} 1,586$, de Las Yayitas (en S11 y S14) y $\widehat{\jmath} 714$, de Loma Las Yayas (en S14). Presencia de callo porífero en todos los ejemplares, la fórmula porífera no varía. Los nódulos presentes en metaterguitos, en general, son pequeños, a veces solo insinuados, su presencia varía entre segmentos y ejemplares, insinuándose en dos hileras a lo largo de margen posterior en segmentos posteriores. Antenas ligeramente más largas en machos, sobrepasando margen posterior de S3 cuando se extienden hacia atrás en algunos machos $(1,584 ; 1,588 ; 1,592$ y 1,595 de Las Yayitas, y en 714 de Loma Las Yayas). En ninguna de las hembras las antenas sobrepasaron margen posterior de S3 al extenderse. Los antenómeros, en general, son gruesos y pubescentes. Epiprocto ligeramente elongado, sin variación notable.

Vista ventral. Tubérculo cónico pequeño en coxas del segundo par de patas en todos los machos examinados. Patas rojas, perdiendo color de los primeros podómeros una vez en alcohol en varios de los ejemplares. En especímenes de Loma Los Pinos (ððત 717 y 718) y Loma Las Yayas (đ 714), las patas se observaron menos rojas al ser colectados. Pleuritos generalmente más oscuros que esternitos. Hipoprocto subtriangular, sin variaciones notables, valvas anales más oscuras y ligeramente estriadas.

Etimología. Epíteto específico en honor a la entomóloga dominicana Ruth Bastardo, entusiasta colaboradora en favor del estudio de los milpiés, figurando entre los primeros colectores de esta especie.

Comentarios. Achromoporus bastardoae sp. nov. resulta de fácil colecta, ya que se observa marchando activamente sobre el camino entre Las Yayitas y Cañaveral, este conspicuo comportamiento lo hace similar a Achromoporus heteromus Loomis, observado y colectado repetidas veces por quien escribe en varias localidades de la provincia San Cristóbal. Entre los milpiés asociados en las localidades de colecta figuran Alcimobolus domingensis (DeSaussure y Humbert) y otro rhinocrícido, así como un quelodésmido, aún no determinados.

Tipos y distribución geográfica. La localidad tipo queda delimitada entre las coordenadas 0358785E - 2035014N, al final del caserío de Las Yayitas (antes del río) y 359969E - 2035734N, subiendo hacia Cañaveral, en Las Yayitas, El Recodo, provincia Peravia, República Dominicana. De la localidad tipo son: MNHNSD Holotipo $\widehat{\jmath} 19.1,590$, paratipos $\widehat{\jmath} \widehat{\jmath} 19.1,584-19.1,588$ (5), paratipos $ᄋ+1$ 19.1,574-19.1,582 (9), al final del caserío, después de El Recodo y antes de cruzar el río, 0358785E-2035014N, 286 metros sobre nivel del mar (msnm), colectados mientras se desplazaban sobre el camino; el paratipo $\lesssim$ 1,587 fue roto para extraer los gonopodos y separar 
sus piezas (se conserva de esta manera). Paratipos đ̋ $\widehat{\jmath} 19.1,592,19.1,593$ y 19.1,595 (3) en camino a Cañaveral, después de cruzar el río, 0358754E-2035422N, 350 msnm, colectados entre piedras y hojarasca; paratipos $+919.1,567,19.1,570-19.1,572,19.1,596$, en el mismo camino hacia Cañaveral, pero entre las coordenadas 359538E-2035433N y 359969E-2035734N, 440$453 \mathrm{msnm}$, colectados mientras se desplazaban sobre el camino. Otros paratipos: MNHNSD

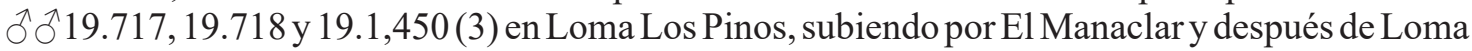
Los Guayuyos, provincia Peravia, 352357E-2035657N, 1,341 msnm, excavando entre piedras y hojarasca. $\lesssim 19.714$, Loma Las Yayas, Honduras, provincia Peravia, 349509E-2034469N, 434 msnm, aproximadamente a tres pulgadas bajo tierra. Colectores: Ruth Bastardo, Gabriel de los Santos, Cristian Marte, Joel González y Carlos Suriel: 27/VI/2009; 26-27/IX/2009; 2-5/VI/2010; 11-12/VIII/2010. Los especímenes fueron depositados en el MNHNSD, excepto los paratipos ${ }^{\top}$ 1,585 y $q 1,581$, donados en intercambio a la colección ARPA.

Achromoporus fractus sp. nov.

Figura 2, A-E

Diagnosis. Gonopodos pequeños. Prefémur corto, proyectado hacia afuera y muy curvado. Acropodito fuertemente curvado desde su base, articulado en ángulo de $90^{\circ}$. Bifurcación del acropodito en su porción media, ambas ramas muy separadas; rama posterior más expandida, aguda en su extremo, rama anterior (solenomerito) mucho más fina en sus porciones media y posterior, con extremo muy agudo y filiforme. Ambas ramas curvadas hacia dentro (fig. 2, C-D). Proceso prefemoral muy grueso desde la base y expandido en su extremo, el cual se hace más agudo al final, pudiendo estar proyectado hacia la región posterior del cuerpo (fig. 2, E). Cuerpo aplanado dorsoventralmente (fig. 2, A-B), más pronunciado en la primera mitad. Color castañorojizo o amarillento dorsalmente, pleuritos castaño oscuro. Dorso sin setas. Pequeño diente en AAP a partir de S2 o S3, a veces ausente en segmentos posteriores. APP agudo e inclinándose hacia atrás progresivamente, menos pronunciado en hembras. Margen posterior de paraterguitos liso. Callos poríferos presentes, abriendo lateralmente y hacia arriba, fórmula porífera normal (5, 7, 9-10, 12-13, 15-19), sin diferencia de color entre segmentos poríferos y no poríferos.

Diagnosis. Gonopods small. Prefemur short, projecting outwards and very curved. Acropodite strongly curved from lower portion, forming a $90^{\circ}$ angle with prefemur, bifurcated from the middle. Both branches very separated; posterior branch wider with apex acute, anterior branch (solenomere) much thinner at central and posterior portions, apex filiform. Both branches curved inwards (fig. 2, C-D). Prefemoral process very thick from its lower portion, wider distally, with apex acute and projecting, sometimes, towards the posterior portion of the body (fig. 2, E). Body dorso-ventrally compressed (fig. 2, A-B), principally at anterior segments. Color reddish chestnut or yellowish at dorsum, pleurites dark chestnut. Dorsum glabrous. Small tooth at PAA from S2 or S3, sometimes absent at posterior segments. PPA acute, inclining backwards progressively, more in males than females. Posterior margin of paraterguites smooth. Peritremata with ozopore opening laterally and upwards, pore formula normal $(5,7,9-10,12-13,15-19)$, poriferous and non-poriferous segments with similar color pattern.

Descripción del Holotipo. Macho (ठ̋). Número catalográfico: MNHNSD 19.1,251 Longitud= $18.2 \mathrm{~mm}$, anchura= $2.8 \mathrm{~mm}$.

Gonopodos. Pequeños, apenas alcanzan región posterior de S6 cuando se extienden. Prefémur corto, muy curvado y proyectado hacia fuera. Acropodito también curvado desde la base, formando ángulo de $90^{\circ}$ con el prefémur, se bifurca en sus dos ramas desde porción media, ambas ramas muy separadas, la posterior más expandida y aguda en su extremo, rama anterior mucho más fina en sus porciones media y posterior, con extremo muy agudo y filiforme. Ambas ramas curvadas hacia dentro (fig. 2, C-D). Proceso prefemoral muy grueso desde la base, 
con extremo expandido y curvado hacia dentro, haciéndose más agudo al final (fig. 2, E).

Vista dorsal. Aplanado, principalmente en la primera mitad del cuerpo. Color castañoamarillento, más claro en metaterguitos. Ausencia de setas y nódulos en los terguitos. Pequeño diente en AAP (S2-S7), insinuado en S8-S12. APP ligeramente más claro que el resto del terguito, agudo, obviamente inclinado hacia atrás a partir de S6, pero más pronunciado desde S11. Callos poríferos abriendo lateralmente y hacia arriba, fórmula porífera normal, sin diferencia de color entre segmentos poríferos y no poríferos.
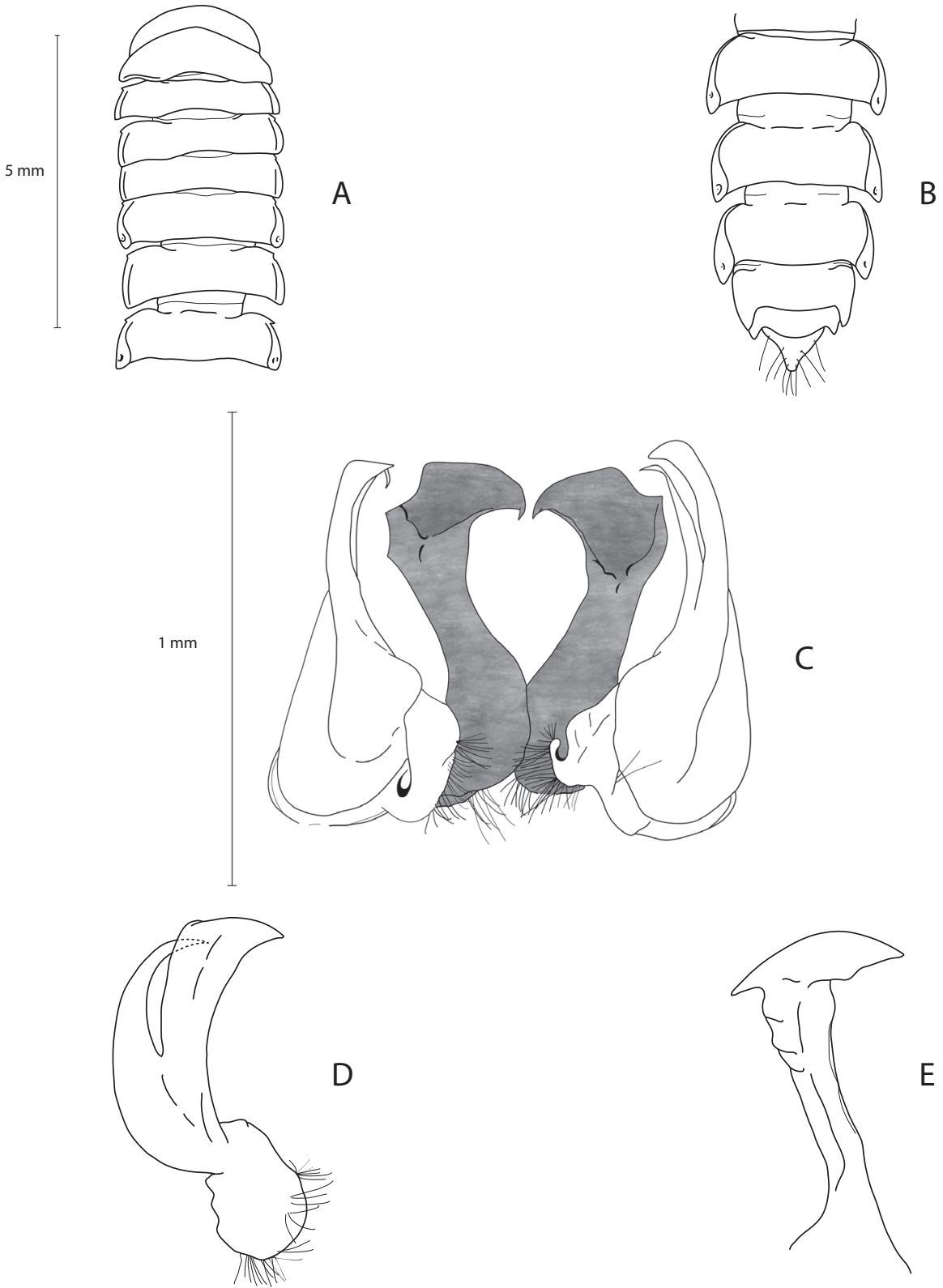

Figura 2. Partes del cuerpo y gonopodos de Achromoporus fractus sp. nov. A, región anterior del cuerpo. B, región posterior del cuerpo. C, gonopodos completos, vista posterior (el proceso prefemoral fue sombreado para diferenciarlo). $\mathrm{D}$, rama gonopodal derecha, vista lateral, girada; la rama posterior translúcida permite ver el extremo de la rama anterior detrás. E, proceso prefemoral, vista antero-lateral. A-C, holotipo, macho 19. 1,251. D-E, paratipo, macho 19. 1,256 . 
Margen posterior de paraterguitos liso. Epiprocto del mismo color que el dorso, ligeramente alargado y puntiagudo, con macrosetas presentes. Antenas delgadas, alcanzan margen posterior de S4 cuando se extienden, antenómeros pubescentes.

Vista ventral. Patas amarillentas, pequeña proyección cónica en coxas del segundo par. Esternitos castaño-rojizos, pleuritos más oscuros. Hipoprocto del mismo color que esternitos, valvas anales más oscuras y ligeramente estriadas.

Variabilidad y regularidades morfológicas. Machos: longitud = $19.24 \mathrm{~mm}(18.2-19.8 ; \mathrm{n}=5)$, anchura $=2.64 \mathrm{~mm}(2.5-2.8 ; \mathrm{n}=5)$. Hembras: longitud $=19.19 \mathrm{~mm}(15.5-22.1 ; \mathrm{n}=12)$, anchura $=2.56 \mathrm{~mm}(2.0-2.9 ; \mathrm{n}=12)$.

Vista dorsal. Cuerpo aplanado en su primera mitad, pero menos en las hembras, las que son ligeramente globosas en la segunda mitad. El color varía entre castaño-rojizo y castaño-amarillento, más claro en metaterguitos; $+1,269$ con metaterguito S8 oscuro. Antenas regularmente delgadas, con antenómeros pubescentes; su longitud es variable pero en ningún caso sobrepasan margen anterior de S5 cuando se extienden. Terguitos sin setas, con nódulos muy pequeños de presencia variable, a veces solo insinuados: $\widehat{\jmath} 1,252$ (insinuados en S5-S7), §̂1,253 (insinuados en S14 y $\mathrm{S} 16$ ), + 1,262 (insinuados en paraterguitos de S6-S8 y S11), ㅇ 1,264 (insinuados en paraterguitos S4-S6), $+1,269$ (insinuados en paraterguitos de S2, S10-S13), §̂1,443 (insinuados en S9-S12 y en S15). Pequeño diente en AAP regularmente en S2-S7, a partir de S7 su presencia es variable hasta S13 (de S3 a S7 en $\precsim 1,443$ ). APP del mismo color que el resto del terguito o ligeramente más claro, agudo, obviamente inclinado hacia atrás en segmentos posteriores, principalmente en los machos. Callo porífero presente y fórmula porífera normal en todos los especímenes examinados. Epiprocto sin variación, ligeramente alargado y puntiagudo, con macrosetas y del mismo color que el dorso.

Vista ventral. Esternitos castaño-rojizos o castaño-amarillentos, pleuritos más oscuros. Patas amarillentas, en algunos especímenes castaño-rojizo en primeros podómeros. Hipoprocto subtriangular, del mismo color que esternitos, valvas anales más oscuras y ligeramente estriadas. Pequeña proyección o tubérculo cónico en coxas del segundo par de patas de los machos.

Etimología. Epíteto específico puesto en referencia a la fragilidad de los milpiés de esta especie: son animales relativamente pequeños y delgados, sus patas se desprenden con facilidad y su cuerpo es débil, muy quebradizo una vez muertos.

Comentarios. Casi todos los ejemplares de Achromoporus fractus sp. nov. fueron colectados bajo la sombra y entre raíces de arbustos del género Myrica, donde se encontraron concentrados en grandes cantidades, excepto los pocos especímenes colectados bajo hojarasca y entre piedras en Loma Los Pinos. No se observó ningún ejemplar caminando fuera de este refugio natural de un remanente de bosque nublado muy antropizado. Los milpiés asociados corresponden a otra especie de la familia Chelodesmidae (nueva, en proceso de descripción), Prostemiulus sp. y Spirobolellus sp., no determinados.

Tipos y distribución geográfica. Localidad tipo: Loma Los Guayuyos, Manaclar, provincia Peravia, República Dominicana. Coordenadas: 0354037E-2036648N. Altitud: 1,359 msnm.

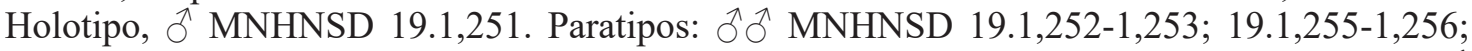
우우 MNHNSD 19.1,261-1,264; 19.1,267-1,269; 19.1,271; 19.1,347-1,349. Otros paratipos: $\lambda$ MNHNSD 19.1,443, q MNHNSD 19.1,444, Loma Los Pinos, Manaclar, provincia Peravia. El paratipo $\widehat{\jmath} 1,256$ fue roto para extraer sus gonopodos y separar las piezas (así se conserva). 
Colectores: Gabriel de los Santos y Carlos Suriel: 28-29/VIII/2009; 25-27/IX/2009. Los especímenes referidos han sido depositados en el MNHNSD, excepto los paratipos $\precsim 1,443 \mathrm{y}$ q 1,263, donados en intercambio a la colección ARPA.

\section{AGRADECIMIENTOS}

Agradecemos al Ministerio de Educación Superior, Ciencia y Tecnología (MESCyT), bajo la dirección de la Maestra Ligia Amada Melo, por hacer posibles las expediciones en las que se colectaron los especímenes estudiados, a través de dos proyectos de investigación con financiamiento del Fondo Nacional de Innovación y Desarrollo Científico y Tecnológico (FONDOCYT) de dicho ministerio. Sean extensibles los agradecimientos a Carlos Rodríguez, Director de Fomento a la Investigación Científica y Tecnológica (MESCyT) y Ruth Bastardo, investigadora principal de los dos proyectos referidos bajo la responsabilidad del Instituto de Investigaciones Botánicas y Zoológicas Rafael M. Moscoso (IIBZ-UASD): "Diversidad biológica de un área natural no protegida: Honduras-El Matadero, Prov. Peravia. Propuesta para la valoración y aprovechamiento sostenible de sus recursos naturales" (proyecto FONDOCYT No. 2008-2-C2-040) y "Factores biológicos y geoclimáticos que modelan las comunidades de moluscos y artrópodos terrestres en dos bosques nublados de la República Dominicana" (proyecto FONDOCYT No. 2009-102); así mismo, para Altagracia Espinosa, coordinadora de ambos proyectos.

Manuel Valdez (IIBZ-UASD) y Brian Farrell (MCZ, Harvard University) facilitaron equipos fotográficos y de microscopía, así como el programa de procesamiento para las fotos, del convenio UASD-Harvard University. Gabriel de los Santos tomó las fotografías al microscopio que sirvieron al autor para los dibujos e hizo correcciones al texto en inglés de las Diagnosis, siendo, además, colaborador en los trabajos de campo. Antonio R. Pérez-Asso revisó el manuscrito e hizo importantes sugerencias. Celeste Mir, Directora del Museo Nacional de Historia Natural de Santo Domingo, apoyó las expediciones e hizo correcciones de estilo al texto en inglés del Abstract y las Diagnosis. Yurkidia Díaz Féliz trabajó la edición de los dibujos con los programas Photoshop CS5 e Ilustrator CS5. David Hernández Martich, Santo Navarro y Cristian Marte ofrecieron su respaldo en los trabajos de campo. Los señores Arsenio González, su hijo Joel González (en Las Yayitas) y Ángel Peguero (en Manaclar) colaboraron con su hospitalidad en el transcurso de las expediciones. De todos nos sentimos muy agradecidos.

\section{LITERATURA CITADA}

Hoffman, R. L. 1979. Clasification of the Diplopoda. Mem. Mus. His. Nat. Geneve. 209 pp.

Hoffman, R. L. 1999. Checklist of the millipedes of North and Middle America. Virg. Mus. Nat. Hist. Spec. Public., 8: 288.

Jeekel, C. A. W. 1971. Nomenclator generum et familiarum Diplopodorum: A list of the genus and family-group names in the Class Diplopoda from the $10^{\text {th }}$ edition of Linnaeus 1758 , to the end of 1957. Nederl. Ent. Ver., Amsterdam, 5: 267 (en línea): http://www.biologie.uniulm.de/systax/, citado febrero, 2011.

Loomis, H. F. 1936. The millipeds of Hispaniola, with descriptions of a new family, new genera, and new species. Bull. Mus. Comp. Zool., 80 (1): 3-197.

Loomis, H. F. 1941. Millipeds collected in Puerto Rico and the Dominican Republic by Dr. P. J. Darlington in 1938. Bull. Mus. Comp. Zool., 88 (2): 17-80. 
Pérez-Asso, A. R. 1996. Revisión del género Amphelictogon (Diplopoda: Polydesmida:

Chelodesmidae) en Cuba. Insecta Mundi, 10: (1-4), 181-216.

Pérez-Asso, A. R. 2005. Dos especies y una subespecie nueva de milpiés del género Achromoporus (Diplopoda: Polydesmida) para Hispaniola. Solenodon, 5: pp. 53-59.

Pérez-Asso, A. R. 2009. El género Achromoporus (Diplopoda: Polydesmida: Chelodesmidae) en República Dominicana: especies nuevas y sinonimias. Solenodon, 8: 33-81.

Pérez-Asso, A. R. y D. E. Pérez-Gelabert 2001. Checklist of the millipeds (Diplopoda) of Hispaniola. Boletín S.E.A. 28: 67-80.

Pérez-Gelabert, D. E. 2008. Arthropods of Hispaniola (Dominican Republic and Haiti): A checklist and bibliography. ZOOTAXA 1831, 71, pp. 530.

Suriel, C. 2010. Dos especies nuevas del género Achromoporus (Diplopoda: Polydesmida: Chelodesmidae) del Parque Nacional José del Carmen Ramírez. Novitates Caribaea, 3, 13-21. 